\title{
Powerful vascular protection by combining cilnidipine with valsartan in stroke-prone, spontaneously hypertensive rats
}

\begin{abstract}
Shinji Takai ${ }^{1}$, Denan Jin ${ }^{1}$, Shizuka Aritomi ${ }^{2}$, Kazumi Niinuma ${ }^{2}$ and Mizuo Miyazaki ${ }^{1}$
Cilnidipine is an L- and N-type calcium channel blocker (CCB), and amlodipine is an L-type CCB. Valsartan (10 $\left.\mathrm{mg} \mathrm{kg}^{-1}\right)$, valsartan $\left(10 \mathrm{mg} \mathrm{kg}^{-1}\right)$ and amlodipine $\left(1 \mathrm{mg} \mathrm{kg}^{-1}\right)$, and valsartan $\left(10 \mathrm{mg} \mathrm{kg}^{-1}\right)$ and cilnidipine $\left(1 \mathrm{mg} \mathrm{kg}^{-1}\right)$ were administered once daily for 2 weeks to stroke-prone, spontaneously hypertensive rats (SHR-SPs). Blood pressure was significantly reduced by valsartan, and it was further reduced by the combination therapies. Vascular endothelial dysfunction was significantly attenuated in all therapeutic groups, and further significant attenuation was observed in the valsartan + cilnidipine-treated group, but not in the valsartan + amlodipine-treated group. Vascular nicotinamide adenine dinucleotide phosphate (NADPH) oxidase subunit NOX1 gene expression was significantly attenuated in all therapeutic groups, and significantly greater attenuation was observed in the valsartan + cilnidipine-treated group than in the valsartan-treated group. Compared with the valsartan-treated group, the positive areas for 4-hydroxy-2-nonenal were significantly lower only in the valsartan + cilnidipinetreated group. Plasma renin activity was significantly augmented in the valsartan-treated group, and it was significantly attenuated in the valsartan + cilnidipine-treated group. A significant increase in the ratio of plasma angiotensin-(1-7) to angiotensin II was observed only in the valsartan + cilnidipine-treated group. Vascular angiotensin-converting enzyme (ACE) gene expression was significantly attenuated only in the valsartan + cilnidipine-treated group, but ACE2 gene expression was significantly higher in all of the therapeutic groups. Thus, valsartan and cilnidipine combination therapy might have a powerful protective effect in the vascular tissues via increases in the angiotensin-(1-7)/angiotensin II ratio in plasma. Hypertension Research (2013) 36, 342-348; doi:10.1038/hr.2012.187; published online 29 November 2012
\end{abstract}

Keywords: angiotensin II receptor blocker; calcium channel blocker; NADPH oxidase; oxidative stress; vascular function

\section{INTRODUCTION}

It is important to lower blood pressure in patients with hypertension to decrease hypertensive complications such as stroke, cardiac failure and renal failure. These complications are closely related to vascular damage, along with endothelial dysfunction. Endothelial dysfunction is thought to mainly depend on the augmentation of oxidative stress in the vasculature. Mechanical stress by high blood pressure augments oxidative stress in the vasculature, and lowering blood pressure reduces oxidative stress. L-type calcium channel blockers (CCBs) are useful for lowering blood pressure and are widely used as antihypertensive agents. L-type CCBs are thought to be useful for attenuation of oxidative stress via reduction of mechanical stress. On the other hand, angiotensin II blockade not only reduces blood pressure, but also shows a powerful vascular protective effect. ${ }^{1,2}$ Although an increase in angiotensin II stimulation via angiotensin II type $1\left(\mathrm{AT}_{1}\right)$ receptor stimulation induces hypertension that indirectly augments oxidative stress via increased mechanical stress, it also directly augments oxidative stress by NADPH oxidase activation via $\mathrm{AT}_{1}$ receptor stimulation. Blockade of angiotensin II function reduces oxidative stress powerfully via attenuation of angiotensin II-augmented oxidative stress, in addition to lowering blood pressure. Therefore, an L-type CCB and angiotensin II receptor blocker (ARB) combination may be useful for powerful vascular protection via strong attenuation of oxidative stress in the vasculature compared with monotherapy with each alone. ${ }^{3-5}$

Voltage-dependent calcium channel subtypes, L-, N-, T- and P/Qtype, are present within the vasculature. Dual blockades of L- and $\mathrm{N}$-type calcium channels and L-and T-type calcium channels may have greater renoprotective effects than mono blockade of L-type calcium channels. Although L-type CCBs have a small effect in glomerular efferent arterioles, where L-type calcium channels are poorly expressed, $\mathrm{L}$ - and N-type CCBs and L-and T-type CCBs dilate both the afferent and efferent arterioles. ${ }^{6}$ These different effects between mono- and dual-channel blockade on the efferent arterioles result in superior renal protection with dual-channel blockade than with mono-channel blockade, both in patients and in experimental models. ${ }^{7-10}$

${ }^{1}$ Department of Pharmacology, Osaka Medical College, Takatsuki City, Osaka, Japan and ${ }^{2}$ Department of Pharmacology, Exploratory Research Laboratories, Research Center, Ajinomoto Pharmaceuticals, Kawasaki, Kanagawa, Japan

Correspondence: Dr Shinji Takai, Department of Pharmacology, Osaka Medical College, 2-7 Daigaku-machi, Takatsuki City, Osaka 569-8686, Japan.

E-mail: pha010@art.osaka-med.ac.jp

Received 5 April 2012; revised 6 September 2012; accepted 12 September 2012; published online 29 November 2012 
$\mathrm{N}$-type calcium channels are predominantly distributed in the sympathetic nervous system and regulate the release of noradrenaline from sympathetic nerve endings. ${ }^{11}$ Cilnidipine is an L- and N-type $\mathrm{CCB}$, and it has shown cardiorenal protection in patients with hypertension. ${ }^{8,12}$ However, it has been unclear whether combination therapy with an L- or N-type CCB and an ARB is useful for preventing vascular dysfunction. In the present study, the effect of L-type CCB + ARB therapy was compared with that of L- and N-type $\mathrm{CCB}+\mathrm{ARB}$ therapy on vascular function in stroke-prone, spontaneously hypertensive rats (SHR-SPs).

\section{METHODS}

\section{Animals}

Eight-week-old male Wistar-Kyoto (WKY) rats and SHR-SPs were obtained from Japan SLC (Shizuoka, Japan). All rats were given normal rat chow (F-2, Funabashi Farm, Chiba, Japan). The experiments were conducted in accordance with the Guide for the Care and Use of Laboratory Animals (Animal Research Laboratory, Osaka Medical College, Osaka, Japan).

Eight-week-old SHR-SPs were orally given placebo $(n=6)$, valsartan $\left(10 \mathrm{mg} \mathrm{kg}^{-1}\right.$ per day) $(n=6)$, valsartan $\left(10 \mathrm{mg} \mathrm{kg}^{-1}\right.$ per day $)+$ amlodipine $\left(1 \mathrm{mg} \mathrm{kg}^{-1}\right.$ per day) $(n=6)$, or valsartan $\left(10 \mathrm{mg} \mathrm{kg}^{-1}\right.$ per day $)+$ cilnidipine $\left(1 \mathrm{mg} \mathrm{kg}^{-1}\right.$ per day) $(n=6)$ by gavage for 2 weeks. In the placebo-treated group, SHR-SPs received the same volume of water as vehicle. Systolic blood pressure (SBP) was monitored by tail-cuff plethysmography (BP-98, Softron, Tokyo, Japan) 1 and 2 weeks after starting the treatment. WKY rats were used as a normal control group $(n=6)$. The rats were anesthetized with $35 \mathrm{mg} \mathrm{kg}^{-1}$ of sodium pentobarbital and sacrificed to obtain blood and tissues, 2 weeks after starting the treatment.

Vascular relaxation in the isolated carotid artery

Isolated rat carotid arteries were cut into $10 \times 1.0-\mathrm{mm}$ helical strips and placed on a myograph under a resting tension of $1.0 \mathrm{~g}$ in Tyrode's solution $\left(137 \mathrm{mmoll}^{-1} \mathrm{NaCl}, 2.7 \mathrm{mmoll}^{-1} \mathrm{KCl}, 1.8 \mathrm{mmoll}^{-1} \mathrm{CaCl}_{2}, 1.1 \mathrm{mmoll}^{-1}\right.$ $\mathrm{MgCl}_{2}, 0.42 \mathrm{mmoll}^{-1} \mathrm{NaH}_{2} \mathrm{PO}_{4}, 12 \mathrm{mmoll}^{-1} \mathrm{NaHCO}_{3}$, and $5.7 \mathrm{mmoll}^{-1}$ glucose, $\mathrm{pH} 7.4$ ) at $37^{\circ} \mathrm{C}$ and continuously bubbled with $5 \% \mathrm{CO}_{2}$ in $\mathrm{O}_{2}{ }^{4,17}$ The strips were initially vasoconstricted with $50 \mathrm{mmoll}^{-1} \mathrm{KCl}$, and then the bathing medium was washed out. Relaxation induced by acetylcholine was assessed after contraction to steady-state tension using $1 \mu \mathrm{moll}^{-1}$ noradrenaline. ${ }^{13}$

\section{Real-time polymerase chain reaction (RT-PCR)}

To measure the gene expressions of several parameters of the aorta, specimens were immediately frozen, and the remaining aorta was fixed for histological assessment. The total RNA $(1 \mu \mathrm{g})$ of the aorta was transcribed into cDNA with Superscript III reverse transcriptase and random hexamers (Invitrogen, Carlsbad, CA, USA). ${ }^{14}$ The mRNA was measured by RT-PCR on a LightCycler with software (Roche Diagnostics, Tokyo, Japan) using TaqMan fluorogenic probes. All primers and probes for RT-PCR of ACE and ACE2, endothelial nitric oxidase synthase (eNOS), nicotinamide adenine dinucleotide phosphate (NADPH) oxidase 1 (NOX1), voltage-dependent N-type calcium channel $\left(\mathrm{Ca}_{\mathrm{V}} 2.2\right)$ and GAPDH were designed by Roche Diagnostics. The sequences of the PCR primers for amplification were as follows: ACE, sense, 5 'TCTGCTTCCCCAACAAGACT-3' and antisense, 5'-AGGAAGCCAGGATGTT
GGT-3'; ACE2, sense, 5'-TCAAGGGAAAAGAACCAGACA-3' and antisense, 5'-GGTTTCAAATCACTCACCCATAC-3'; eNOS, sense, 5'-TGACCCTCACC GATACAACA-3' and antisense, 5'-CGGGTGTCTAGATCCATGC-3'; NOX1, sense, 5'-GGCATCCCTTTACTCTGACCT- $3^{\prime}$ and antisense, 5'-TGCTGCTCG AATATGAATGG-3'; Ca 2.2 , sense, $5^{\prime}$-TAAGCGCATCACCGAATG- $3^{\prime}$ and antisense, 5'-GGCCAGGACAATACAGTTGG-3'; and for GAPDH, sense, 5'AATGTATCCGTTGTGGATCTGA-3' and antisense, 5'-GCTTCACCACCTCTT GATGT-3'. The probes for amplification were as follows: ACE, $5^{\prime}$-GACCCAGA-3'; ACE2, 5'-GGAGGCAG-3'; eNOS, 5'-TGTGGCTG-3; NOX1, 5' -CAGAGGAA-3'; $\mathrm{Ca}_{\mathrm{V}} 2.2,5^{\prime}$-GGCCACCA-3'; and GAPDH, $5^{\prime}$-CCTGGAGA- ${ }^{\prime}$. The mRNA levels of ACE, ACE2, eNOS and NOX1 were normalized to that of GAPDH.

\section{Immunohistochemistry}

Fixed aortic tissues were embedded in paraffin, and then each block was sectioned at a thickness of $3 \mu \mathrm{m}$. The sections were incubated with $3 \% \mathrm{H}_{2} \mathrm{O}_{2}$ in methanol to inhibit endogenous peroxidase, then incubated with proteinblocking solution (Dako, Tokyo, Japan) to block non-specific antigens. For immunostaining against 4-hydroxy-2-nonenal (4-HNE), sections were incubated with mouse 4-HNE antibody (Nikken Seil, Shizuoka, Japan). ${ }^{14}$ A secondary biotinylated anti-IgG antibody (Invitrogen) was used. Sections were visualized using horseradish peroxidase and 3-amino-9-ethylcarbozole as the substrate-chromogen (Dako). ${ }^{14}$ The nuclei were counterstained using hematoxylin. The immunostain-positive area was quantified with an image analysis system (model VM-30, Olympus Optical, Tokyo, Japan).

\section{Measurements of biochemical parameters in plasma}

Plasma was separated from the blood samples by centrifugation at $3000 \mathrm{~g}$ for $15 \mathrm{~min}$ at $4{ }^{\circ} \mathrm{C}$. Plasma renin activity (PRA) was measured by standard radioimmunoassay methods (Mitsubishi Chemical Medience, Tokyo, Japan). Plasma angiotensin II concentrations were measured using an enzyme immunoassay kit (Peninsula Laboratories, Belmont, CA, USA). Plasma angiotensin-(1-7) concentrations were purified via solid-phase extraction and measured using liquid chromatography tandem mass spectrometry (3200 QTRAP LC/MS/MS, AB SCIEX, Tokyo, Japan).

\section{Statistical analysis}

Data are expressed as means \pm s.e.m. Statistical analyses were performed using a parametric test with Fisher's protected least significant difference. Differences were considered significant when the $P$-value was $<0.05$.

\section{RESULTS}

\section{Body weight and heart weight}

Body weight, heart weight and the ratio of the heart weight to body weight are shown in Table 1. Body weight was significantly heavier in the WKY rats than in the placebo-treated SHR-SPs, but there were no significant differences among the SHR-SP groups. Heart weights were not significantly different among the groups. The ratio of heart weight to body weight was significantly higher in the placebo-treated SHRSPs than in the WKY rats. The ratio was significantly lower both in the valsartan + amlodipine- and valsartan + cilnidipine-treated groups than in the placebo-treated group, but no significant difference in the ratio was observed between these two groups.

Table 1 Body weight, heart weight and the ratio of heart weight to body weight

SHR-SP

\begin{tabular}{|c|c|c|c|c|c|}
\hline Parameters & $W K Y$ rats & Placebo $(\mathrm{n}=6)$ & Valsartan $(\mathrm{n}=6)$ & Valsartan + amlodipine $(\mathrm{n}=6)$ & Valsartan + cilinidipine $(\mathrm{n}=6)$ \\
\hline Body weight (g) & $272 \pm 3.0 * *$ & $206 \pm 3.3$ & $204 \pm 4.8$ & $206 \pm 4.2$ & $205 \pm 3.5$ \\
\hline Heart weight (mg) & $768 \pm 18$ & $820 \pm 30$ & $698 \pm 9 *$ & $707 \pm 16^{*}$ & $705 \pm 16^{*}$ \\
\hline Heart weight/body weight (mg/g) & $2.82 \pm 0.07^{* *}$ & $3.98 \pm 0.10$ & $3.43 \pm 0.06 * *$ & $3.43 \pm 0.06 * *$ & $3.44 \pm 0.05^{* *}$ \\
\hline
\end{tabular}

Values are mean \pm s.e.m. ${ }^{*} P<0.05$ and ${ }^{* *} P<0.01$ vs. placebo. 
Blood pressure in SHR-SPs

In SHR-SPs, SBPs were $191 \pm 3.4,191 \pm 3.7,192 \pm 3.4$, and $191 \pm 2.5 \mathrm{~mm} \mathrm{Hg}$ before administration of placebo, with valsartan, with valsartan + amlodipine and with valsartan + cilnidipine, respectively, and in the age-matched WKY rats, SBP was $119 \pm 2.0 \mathrm{~mm} \mathrm{Hg}$ (Figure 1). SBP was $229 \pm 4.1 \mathrm{~mm} \mathrm{Hg}$ after the last placebo treatment, but it was significantly decreased to $183 \pm 1.8 \mathrm{~mm} \mathrm{Hg}$ by valsartan. SBPs in the valsartan + amlodipine- and valsartan + cilnidipinetreated groups were $166 \pm 2.4$ and $168 \pm 2.2 \mathrm{~mm} \mathrm{Hg}$, respectively; both were significantly lower than in the valsartan-treated groups.

\section{Vascular relaxation and gene expressions of eNOS and NOX1}

In all rats, acetylcholine-induced vascular relaxation was observed as an index of endothelial function (Figure 2a). Vascular relaxation was significantly lower in the placebo-treated SHR-SPs than in the WKY rats, and was significantly greater in all valsartan-, valsartan + amlodipine- and valsartan + cilnidipine-treated SHR-SPs than in placebo-treated SHR-SPs (Figure 2a). Of note, vascular relaxation was significantly greater in the valsartan + cilnidipine-treated SHRSPs than in the valsartan-treated SHR-SPs, but no significant difference between the valsartan- and the valsartan + amlodipinetreated SHR-SPs was observed (Figure 2a).

Gene expression of NADPH oxidase subunit NOX1 in the aorta was significantly higher in the placebo-treated SHR-SPs than in the WKY rats (Figure $2 \mathrm{~b}$ ). NOX1 gene expression was significantly lower in all groups treated with valsartan than in the placebo-treated group in SHR-SPs (Figure 2b). A significant attenuation of NOX1 in the valsartan + cilnidipine-treated group was observed compared with that in the placebo-treated group, but there was no significant difference between the valsartan- and valsartan + amlodipine-treated groups (Figure $2 \mathrm{~b}$ ).

eNOS gene expression in the aorta tended to be lower in the placebo-treated SHR-SPs than in the WKY rats (Figure 2c). eNOS gene expression was significantly higher in all valsartan-, valsartan + amlodipine- and valsartan + cilnidipine-treated SHR-SPs than in the placebo-treated SHR-SPs, and it was significantly higher in the

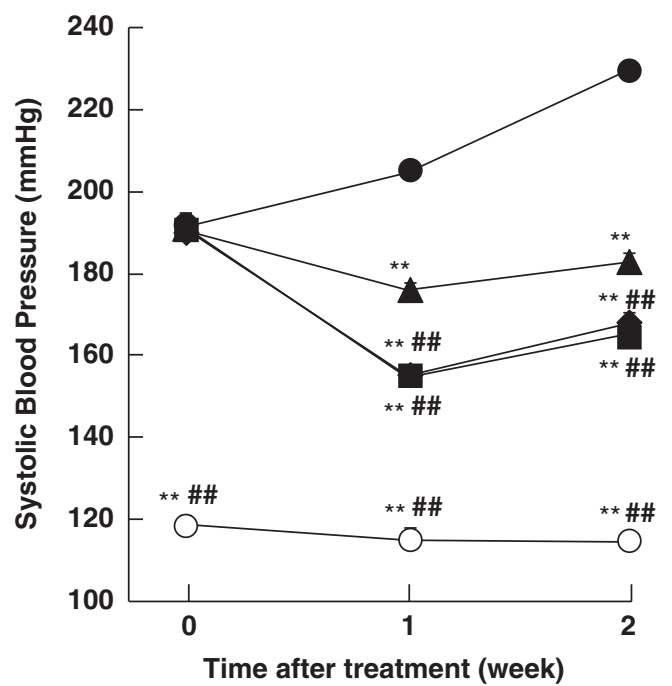

Figure 1 SBP in the WKY rats (open circles) and placebo (closed circles)-, valsartan (closed triangles)-, valsartan + amlodipine (closed squares)- and valsartan + cilnidipine (closed diamonds)-treated SHR-SPs at 0, 1 and 2 weeks after starting the treatment. ${ }^{*} P<0.01$ vs. placebo-treated SHR-SPs. \#\# $P<0.01$ vs. valsartan-treated SHR-SPs. valsartan + cilnidipine-treated SHR-SPs, but not in the valsartan + amlodipine-treated SHR-SPs, than in the valsartan-treated SHR-SPs (Figure 2c).

\section{Vascular immunohistochemistry of 4-HNE}

To evaluate the products of oxidative stress, the 4-HNE-positive area in the media of the aorta was evaluated (Figure 3a). This area was small in the WKY rat, but it was much larger in the placebo-treated SHR-SPs. Although 4-HNE-positive areas were observed in the valsartan-, valsartan + amlodipine- and valsartan + cilnidipine-treated SHR-SPs, they were clearly smaller than in the placebo-treated SHR-SPs. The ratio of the 4-HNE-positive area to the total area in the media of the aorta was significantly higher in the placebo-treated SHR-SPs than in the WKY rats (Figure 3b). Significant attenuation of the ratio was observed in the valsartan-, valsartan + amlodipine- and valsartan + cilnidipine-treated SHR-SPs compared with the placebotreated SHR-SPs (Figure 3b). Furthermore, the ratio was significantly lower in the valsartan + cilnidipine-treated SHR-SPs than in the valsartan-treated SHR-SPs, but no significant difference between the valsartan- and valsartan + amlodipine-treated SHR-SPs was observed (Figure 3b).

\section{Vascular gene expression of N-type calcium channels}

The vascular gene expression of $\mathrm{N}$-type calcium channels was significantly higher in the placebo-treated group than in the normal
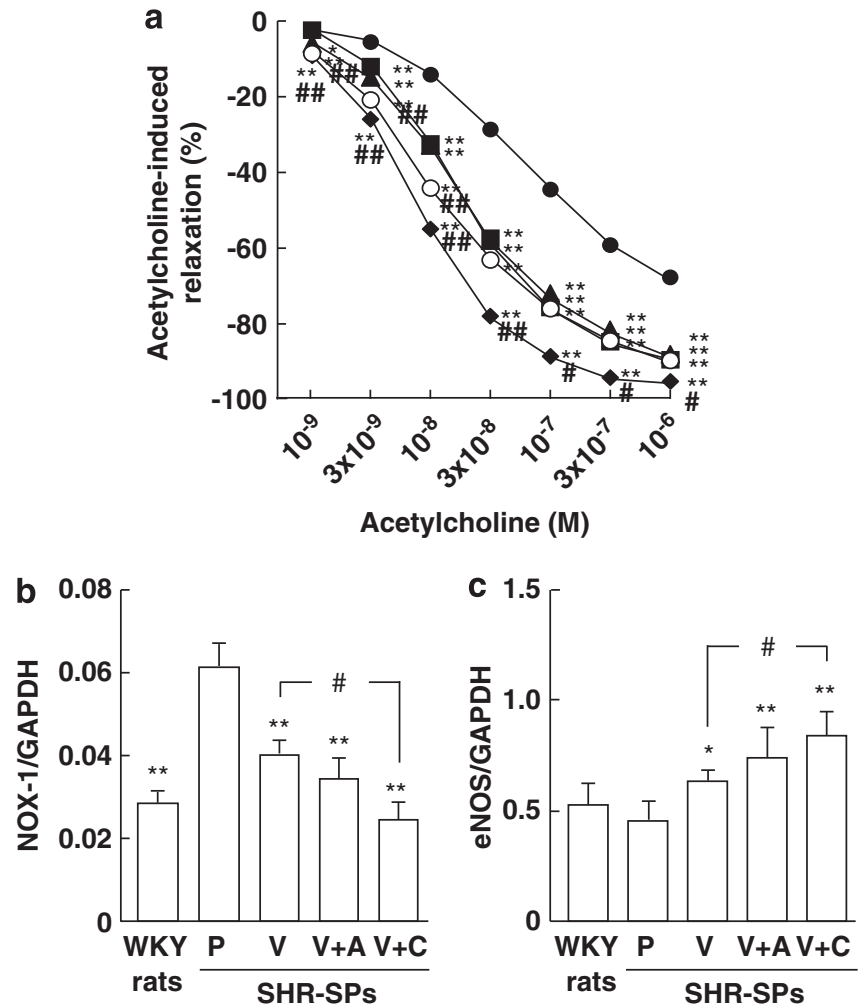

Figure 2 Acetylcholine-induced vascular relaxation in noradrenalineprecontracted carotid arteries in the WKY rats (open circles) and placebo (closed circles)-, valsartan (closed triangles)-, valsartan + amlodipine (closed squares)- and valsartan + cilnidipine (closed diamonds)-treated SHR-SPs (a). Gene expressions of NOX1 (b) and eNOS (c) in aortas obtained from the WKY rats and placebo (P)-, valsartan (V)-, valsartan+amlodipine $(\mathrm{V}+\mathrm{A})$ - and valsartan + cilnidipine $(\mathrm{V}+\mathrm{C})$-treated SHR-SPs. ${ }^{*} P<0.05$ and $* * P<0.01$ vs. placebo-treated SHR-SPs. ${ }^{\#} P<0.05$ vs. valsartan-treated SHR-SPs. 

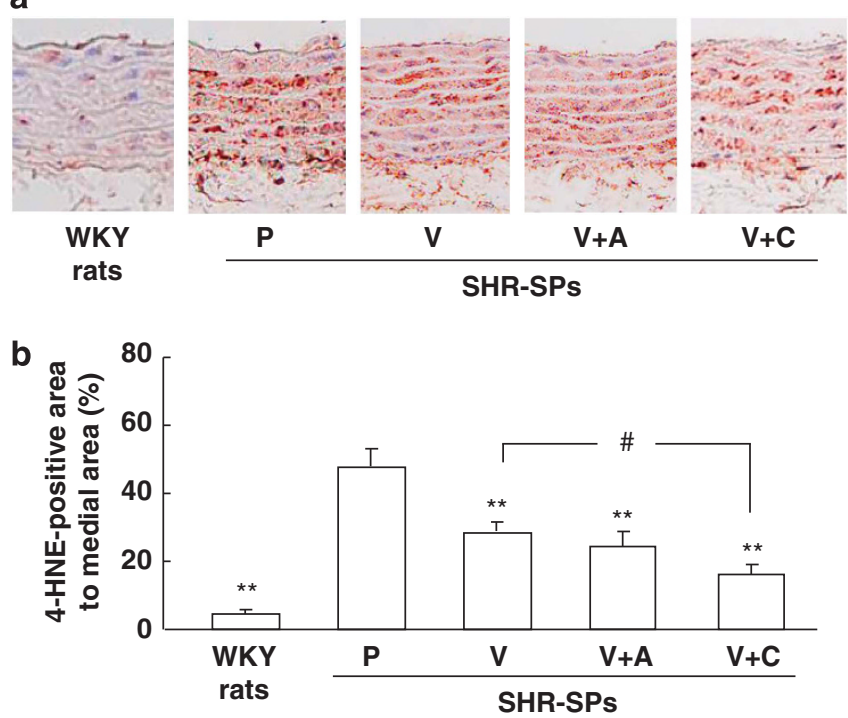

Figure 3 Typical photographs of anti-4-HNE antibody-stained aortas in a WKY rat and placebo-, valsartan-, valsartan + amlodipine- and valsartan + cilnidipine-treated SHR-SPS (a). Ratios of the anti-4-HNE-stained area to the total area in the WKY rats and placebo (P)-, valsartan (V)-, valsartan + amlodipine $(\mathrm{V}+\mathrm{A})$ - and valsartan + cilnidipine $(\mathrm{V}+\mathrm{C})$-treated SHR-SPs (b). ${ }^{*} P<0.01$ vs. placebo-treated SHR-SPs. ${ }^{*} P<0.05$ vs. valsartan-treated SHR-SPs.

group (Figure 4). The gene expressions in the valsartan- and valsartan + amlodipine-treated groups tended to be lower than in the placebo-treated group, although there was no significant difference among these groups (Figure 4). On the other hand, gene expression was significantly lower in the valsartan + cilnidipinetreated group than in both the placebo-treated group and the valsartan-treated group (Figure 4).

\section{Biochemical parameters in plasma}

PRA was significantly higher in all of the therapeutic groups, but it was significantly lower in the valsartan + cilnidipine-treated group than in the valsartan-treated group (Figure $5 \mathrm{a}$ ).

There were no significant differences in the plasma angiotensin II concentration among all groups other than in the valsartan + cilnidipine-treated group (Figure 5b). The plasma angiotensin II concentration was significantly lower in the valsartan + cilnidipinetreated group than in the valsartan-treated group (Figure 5b).

Plasma angiotensin-(1-7) concentration tended to be lower in the placebo-treated group than in the normal group, but there was no significant difference between these two groups (Figure 5c). The plasma angiotensin-(1-7) concentration was significantly higher in the valsartan-treated group than in the placebo-treated group. Further increases of the plasma angiotensin-(1-7) concentrations were observed in these combination-treated groups, but there was no significant difference between the placebo-treated group and all of the therapeutic groups (Figure $5 \mathrm{c}$ ).

The ratio of angiotensin-(1-7) to angiotensin II was significantly lower in the placebo-treated group than in the valsartan-treated group (Figure 5d). The ratio tended to be higher in the valsartan + amlodipine group, but there was no significant difference. On the other hand, the ratio was significantly higher in the valsartan + cilnidipine-treated group than in the valsartan-treated group (Figure 5d).

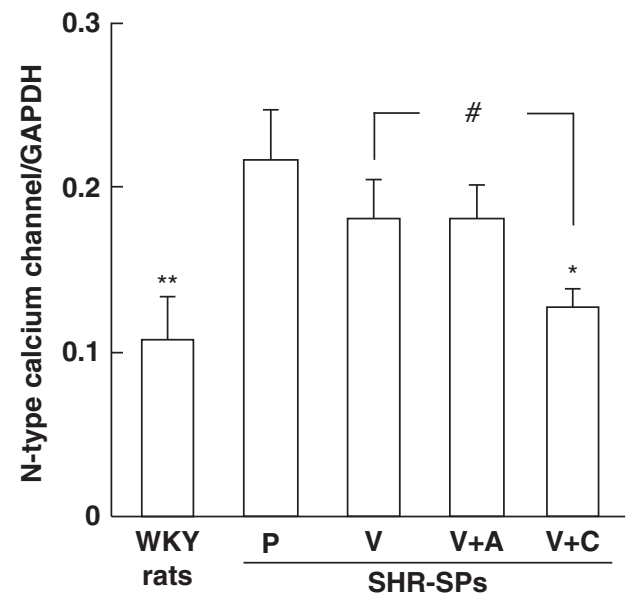

Figure 4 Gene expressions of $\mathrm{N}$-type calcium channels (Cay2.2) in aortas obtained from the WKY rats and placebo (P)-, valsartan (V)-, valsartan + amlodipine $(\mathrm{V}+\mathrm{A})$ - and valsartan + cilnidipine $(\mathrm{V}+\mathrm{C})$-treated SHR-SPs. ${ }^{*} P<0.05$ and ${ }^{* *} P<0.01$ vs. placebo-treated SHR-SPs. $\# P<0.05$ vs. valsartan-treated SHR-SPs.

\section{Vascular gene expressions of ACE and ACE2}

Significant augmentation of ACE gene expression in the aorta was observed in the placebo-treated SHR-SPs compared with the WKY rats (Figure 6a). ACE gene expression was significantly lower in the valsartan + cilnidipine-treated SHR-SPs than in the valsartan + cilnidipine-treated group, but there was no significant difference among the placebo-, valsartan- and valsartan + amlodipine-treated groups (Figure 6a).

Vascular ACE2 gene expression was lower in the placebo-treated SHR-SPs than in the WKY rats (Figure 6b). However, ACE2 gene expression was significantly higher in the valsartan-, valsartan + amlodipine and valsartan + cilnidipine-treated SHR-SPs than in the placebo-treated group, but there was no significant different among these groups (Figure 6b).

\section{DISCUSSION}

In the present study, SBP in 8-week-old SHR-SPs was shown to be about $190 \mathrm{~mm} \mathrm{Hg}$ before treatment, but it increased to $230 \mathrm{~mm} \mathrm{Hg}$ in the placebo-treated group, 2 weeks after starting the experiment. In the valsartan-treated group, SBP was significantly decreased to $183 \mathrm{~mm} \mathrm{Hg}$ at 2 weeks. At the same time, further significant hypotensive effects both in the valsartan + amlodipine- and valsartan + cilnidipine-treated groups were observed compared with the valsartan-treated group, but the hypotensive effect was not significantly different between these two groups. On the other hand, acetylcholine-induced vascular relaxation, which is used as an index of endothelial function, was significantly attenuated in the placebotreated group. The relaxation was significantly augmented by valsar$\tan$ monotherapy, and it was almost the same as that in the normotensive WKY rats. Greater augmentation than with the monotherapy was observed with valsartan and cilnidipine combination therapy, but there was no significant difference between monotherapy and valsartan and amlodipine combination therapy. Dong et al. ${ }^{15}$ demonstrated that 4 weeks' treatment with valsartan and amlodipine provided a significant augmentation of acetylcholine-induced vascular relaxation compared with valsartan treatment in salt-loaded SHR-SPs. In that study, both amlodipine monotherapy and combination therapy with valsartan and amlodipine showed similar hypotensive 

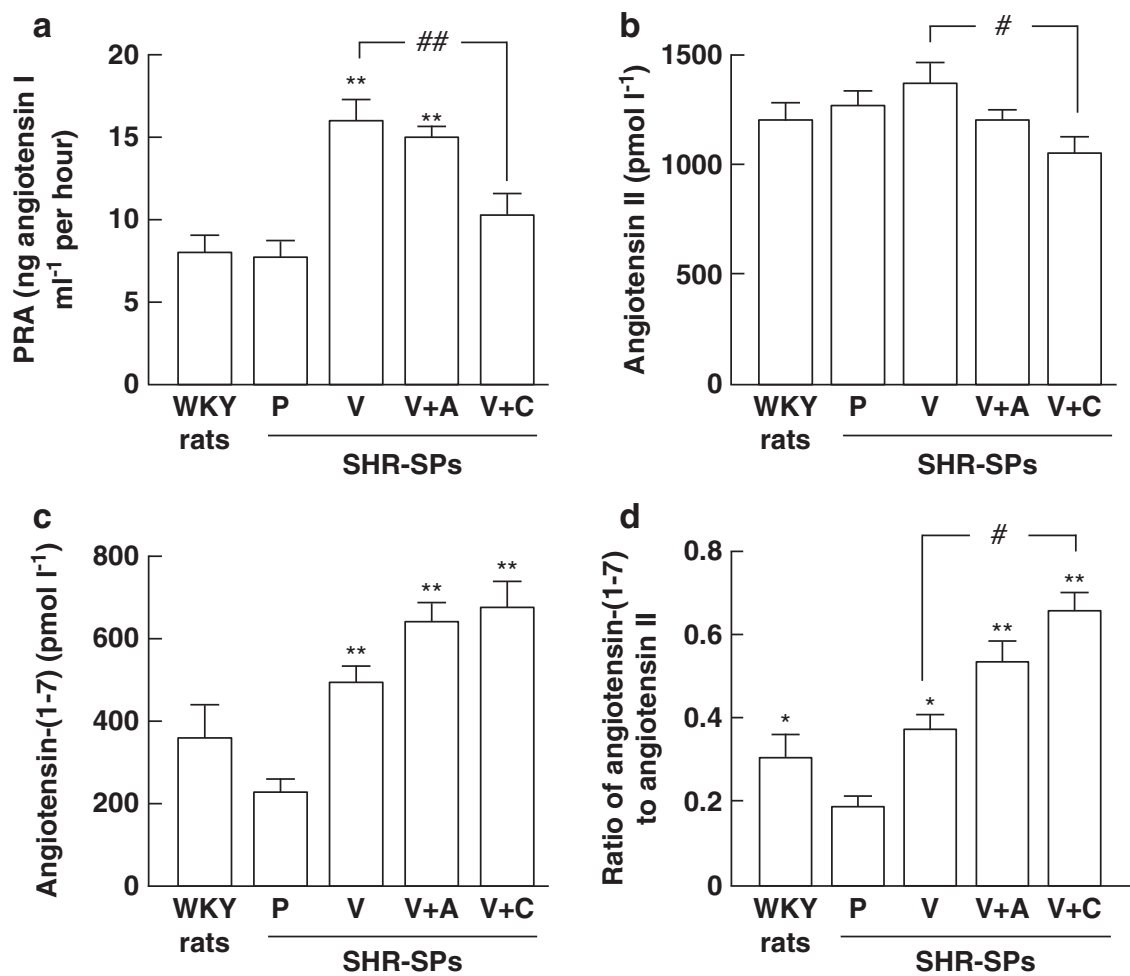

Figure 5 PRA (a), angiotensin II level (b), angiotensin-(1-7) level (c), and the ratio of angiotensin-(1-7) to angiotensin II (d) in plasma obtained from WKY rats and placebo $(\mathrm{P})$-, valsartan $(\mathrm{V})$-, valsartan + amlodipine $(\mathrm{V}+\mathrm{A})$ - and valsartan + cilnidipine $(\mathrm{V}+\mathrm{C})$-treated SHR-SPs. ${ }^{*} P<0.05$ and ${ }^{* *} P<0.01$ vs. placebo-treated SHR-SPs. ${ }^{\#} P<0.05$ and ${ }^{\# \# P}<0.01$ vs. valsartan-treated SHR-SPs.
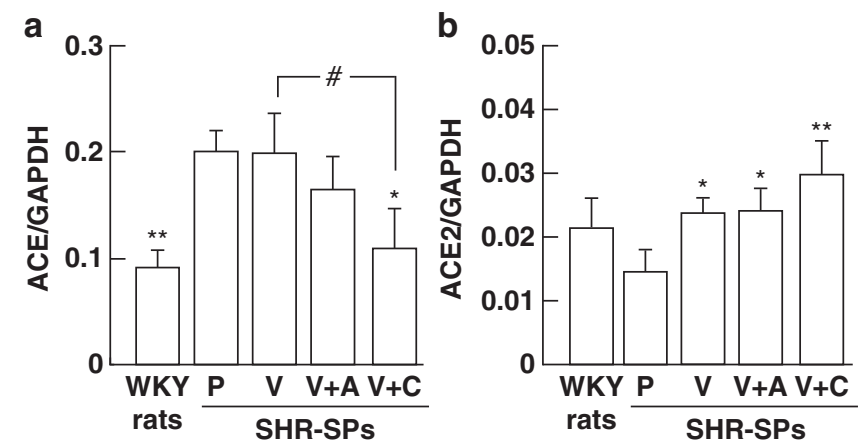

Figure 6 Gene expressions of ACE (a) and ACE2 (b) in aortas obtained from the WKY rats and placebo $(P)-$, valsartan $(V)-$, valsartan + amlodipine $(\mathrm{V}+\mathrm{A})$ - and valsartan + cilnidipine $(\mathrm{V}+\mathrm{C})$-treated SHR-SPs. ${ }^{*} P<0.05$ and $* * P<0.01$ vs. placebo-treated SHR-SPs. $\#<<0.05$ vs. valsartan-treated SHR-SPS.

effects, but the combination therapy also significantly augmented acetylcholine-induced vascular relaxation compared with amlodipine monotherapy in salt-loaded SHR-SPs. ${ }^{15}$ Taken together with the findings of the present study, this may suggest the importance of factors other than the hypotensive effect in conferring vascular protection, such as an anti-oxidative effect. In the present study, we did not observe a beneficial effect of valsartan and amlodipine combination therapy despite the additional hypotensive effect. This might be explained by the short treatment period. In fact, long-term treatment with valsartan and amlodipine significantly prolonged survival time compared with amlodipine monotherapy, despite similar blood pressure. ${ }^{15}$ On the other hand, a mechanism other than hypotensive effect may explain why valsartan and cilnidipine combination therapy showed additional mechanisms for augmentation of vascular relaxation in the short treatment period.

Vascular endothelial function is directly affected by nitric oxide bioavailability, and its dysfunction is associated with oxidative stress. ${ }^{16,17}$ In fact, the 4 -HNE-positive area in vessels was seen as an indicator of oxidative stress, and a significant increase in the placebotreated SHR-SPs was observed in comparison with the WKY rats, which reflected significant attenuation of acetylcholine-induced vascular relaxation in the placebo-treated SHR-SPs. The 4-HNEpositive area observed in placebo-treated SHR-SPs was significantly decreased by treatment with valsartan, and acetylcholine-induced vascular relaxation was significantly augmented. Furthermore, a significant decrease in the 4-HNE-positive area and a significant augmentation of the acetylcholine-induced vascular relaxation were observed with valsartan and cilnidipine combination therapy, but not with valsartan and amlodipine combination therapy, compared with valsartan monotherapy. Thus, the superior attenuation of vascular endothelial dysfunction in valsartan and cilnidipine combination therapy might be dependent on the greater reduction of oxidative stress.

The reason why combination therapy with valsartan and cilnidipine showed greater oxidative stress reduction than valsartan and amlodipine with the same amount of additional hypotensive effect needs to be considered. In the vasculature, NADPH oxidase is known to augment oxidative stress. Vascular gene expression of NADPH oxidase subunit NOX1 was significantly augmented in the placebo-treated group in the present study. Mechanical stress such as high blood pressure acutely activates the NADPH oxidase in vascular smooth 
muscle and endothelial cells. ${ }^{18}$ On the other hand, an increase in NADPH oxidase is closely related to oxidative stress caused by angiotensin II in the vascular tissue of hypertensive rats, and the oxidative stress caused by NADPH oxidase has an important role in the development of vascular dysfunction. ${ }^{19-21}$ In the present study, gene expression of an NADPH oxidase subunit in SHR-SPs was attenuated by valsartan monotherapy, and significant additional attenuation was observed with combination therapy with valsartan and cilnidipine, but not with valsartan and amlodipine. Therefore, the greater attenuation of oxidative stress with valsartan and cilnidipine combination therapy may be closely related to the powerful attenuation of vascular NADPH oxidase.

In the present study, an L-type CCB (amlodipine) or an L- and N-type CCB (cilnidipine) was used in combination with valsartan. L-type and N-type calcium channels are predominantly distributed in vascular smooth muscle cells and sympathetic nerve terminals, respectively. ${ }^{11}$ In cultured human mesangial cells, cilnidipine showed significantly higher antioxidant activity than amlodipine. ${ }^{22}$ Cilnidipine also has a neuroprotective effect via attenuation of oxidative stress in PC12 cells. ${ }^{23}$ In the present study, there was a significant increase of $\mathrm{N}$-type calcium channel gene expression in SHR-SPs. However, this expression was significantly reduced with valsartan treatment, and it was significantly lower again in valsartan and cilnidipine combination therapy than in valsartan monotherapy. In SHRs, an increase in vascular oxidative stress is one factor increasing noradrenaline release from sympathetic nerve terminals, and this augmented noradrenaline release may be also intensify oxidative stress in the vasculature. ${ }^{24,25}$ Cilnidipine may attenuate oxidative stress in vascular tissues by reducing noradrenaline release from sympathetic nerve terminals.

Angiotensin-(1-7) augments vasodilation via increases in bradykinin and nitric oxide release. ${ }^{26}$ This action is opposite to the vasoconstriction mediated by nitric oxide attenuation in response to angiotensin II. Angiotensin-(1-7) is formed by two pathways. In one pathway, angiotensin I is converted to angiotensin II by ACE, and then angiotensin II is converted to angiotensin-(1-7) by ACE2. In the other, angiotensin I is converted to angiotensin-(1-9) by ACE2, and then angiotensin-(1-9) is converted to angiotensin-(1-7) by ACE. ${ }^{27}$ The increase of the ratio of angiotensin-(1-7) to angiotensin II is thought to attenuate a powerful oxidative stress, because angiotensin(1-7) has an antioxidative effect in vascular tissues and angiotensin II has an oxidative effect. ${ }^{28,29}$ In the present study, a significant increase of the ratio of angiotensin-(1-7) to angiotensin II in plasma was observed only in valsartan and cilnidipine combination therapy. Ishiyama et al. ${ }^{30}$ showed that ARBs augmented not only angiotensin II levels, but also angiotensin-(1-7) levels in plasma after myocardial infarction in rats. In SHR, the augmentation of plasma angiotensin-(1-7) levels by ARBs may be dependent on induction of $\mathrm{ACE} 2$ gene expression via $\mathrm{AT}_{1}$ receptor blockade, because angiotensin II downregulates ACE2 gene expression by $\mathrm{AT}_{1}$ receptor stimulation. ${ }^{31,32}$ In fact, a significant augmentation of vascular ACE2 gene expression was observed in all valsartan-treated groups in the present study. On the other hand, vascular ACE expression was significantly augmented in the placebo-treated SHRSP, but ACE gene expression was significantly attenuated by valsartan and cilnidipine combination therapy, but not by valsartan and amlodipine therapy. Toba et al. ${ }^{8}$ demonstrated that cilnidipine, but not amlodipine, attenuated ACE gene expression in the kidney in hypertensive rats. These findings suggest that downregulation of ACE2 by valsartan and upregulation of ACE2 by cilnidipine might contribute to the increase of the ratio of angiotensin-(1-7) to angiotensin II observed in valsartan and cilnidipine combination therapy. Although it has not been confirmed whether angiotensin-(17 ) is predominantly formed from angiotensin II by ACE2 or from angiotensin-(1-9) by ACE, increase in the ratio of angiotensin-(1-7) to angiotensin II was observed in valsartan and cilnidipine combination therapy. This increase might contribute to the superior vascular protective effect of valsartan and cilnidipine combination therapy compared with valsartan and amlodipine combination therapy despite similar hypotensive effect.

The increase in the ratio of angiotensin-(1-7) to angiotensin II in valsartan and cilnidipine combination therapy may be also be involved in other mechanisms. Only with valsartan and cilnidipine combination therapy was the plasma angiotensin II level attenuated significantly in the present study. Both valsartan monotherapy, as well as valsartan and amlodipine combination therapy showed significant augmentation of PRA, but valsartan and cilnidipine combination therapy did not. Cilnidipine alone did not cause attenuation of PRA, but it could attenuate PRA in combination therapy with an ARB in previous studies. ${ }^{33,34}$ Cilnidipine reduces sympathetic nervous system activity, which may result in the reduction of plasma renin release from juxtaglomerular cells through sympathetic nervous system activation. On the other hand, amlodipine did not attenuate the increase in PRA and angiotensin II levels caused by valsartan. Thus, cilnidipine may be useful for counteracting elevation of angiotensin II levels by ARBs, and this action may also accelerate the increase of the ratio of angiotensin-(1-7) to angiotensin II, resulting in the powerful vascular protection observed.

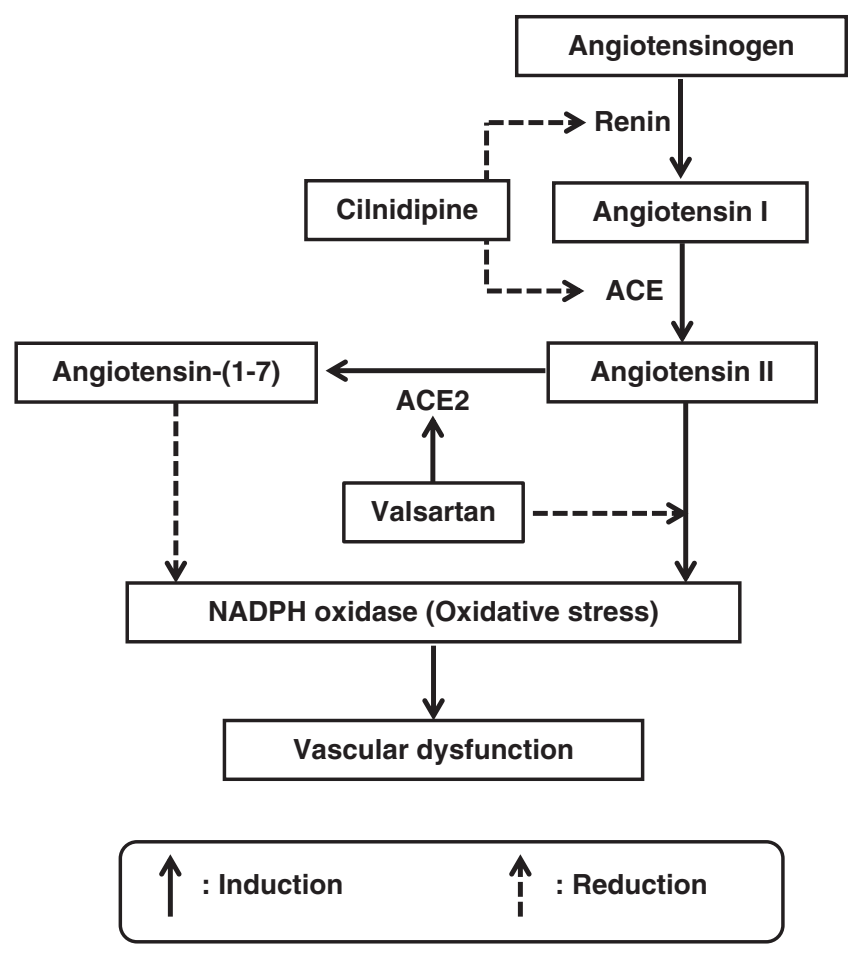

Figure 7 Cilnidipine attenuates angiotensin II formation via inhibition of renin release and ACE activity. Valsartan augments angiotensin-(1-7) formation via induction of ACE2 gene expression. Angiotensin II induces oxidative stress, but, in contrast, angiotensin-(1-7) reduces it. Combination therapy with valsartan and cilnidipine induces upregulation of angiotensin(1-7) and downregulation of angiotensin II, respectively, and these effects result in augmenting the angiotensin-(1-7)/angiotensin II ratio and powerful attenuation of oxidative stress. 
In the present study, eNOS gene expression was significantly higher with valsartan monotherapy and combination therapies with valsartan than with placebo treatment. This phenomenon, that several ARBs showed vascular eNOS gene expression, has also been observed in SHR-SPs. ${ }^{13,17}$ Vascular eNOS gene expression is known to be attenuated by oxidative stress, and the augmentation of eNOS gene expression by valsartan treatment may be related to the reduction of oxidative stress. The further significant augmentation of eNOS gene expression with combination therapy with valsartan and cilnidipine might also be related to the powerful attenuation of oxidative stress. On the other hand, Fan et al. ${ }^{35}$ demonstrated that eNOS gene expression was significantly augmented after treatment with cilnidipine in the human internal thoracic artery. Amlodipine also increases endothelial nitric oxide release by modulating binding of eNOS protein complex to caveolin- $1 .{ }^{36}$ Further studies might be needed to investigate the degree of nitric oxide release caused by monotherapy with amlodipine or cilnidipine.

In conclusion, valsartan and cilnidipine combination therapy showed powerful oxidative stress reduction along with augmentation of the angiotensin-(1-7)/angiotensin II ratio via reduction of angiotensin II by cilnidipine and induction of angiotensin-(1-7) by valsartan (Figure 7). Some clinical studies have shown the superiority of the L- and N-type CCB, cilnidipine, in terms of the improvement of arterial stiffness compared with the L-type CCBs, amlodipine or nifedipine, in patients with hypertension. ${ }^{12,37}$ Combination therapy with an ARB and an L- and N-type CCB may give more useful vascular protection than that with an ARB and an L-type CCB.

\section{CONFLICT OF INTEREST}

The authors declare no conflict of interest.

1 Griendling KK, Minieri CA, Ollerenshaw JD, Alexander RW. Angiotensin II stimulates NADH and NADPH oxidase activity in cultured vascular smooth muscle cells. Circ Res 1994; 74: 1141-1148.

2 Rajagopalan S, Kurz S, Munzel T, Tarpey M, Freeman BA, Griendling KK, Harrison DG. Angiotensin II-mediated hypertension in the rat increases vascular superoxide produc tion via membrane $\mathrm{NADH} / \mathrm{NAD}(\mathrm{P}) \mathrm{H}$ oxidase activation. Contribution to alterations of vasomotor tone. J Clin Invest 1996; 97: 1916-1923.

3 Jinno T, Iwai M, Li Z, Li JM, Liu HW, Cui TX, Rakugi H, Ogihara T, Horiuchi M. Calcium channel blocker azelnidipine enhances vascular protective effects of $\mathrm{AT}_{1}$ receptor blocker olmesartan. Hypertension 2004; 43: 263-269.

4 Inaba S, Iwai M, Tomono Y, Senba I, Furuno M, Kanno H, Okayama H, Mogi M, Higak $\mathrm{J}$, Horiuchi M. Prevention of vascular injury by combination of an $\mathrm{AT}_{1}$ receptor blocker olmesartan, with various calcium antagonists. Am J Hypertens 2009; 22: 145-150.

5 Takai S, Jin D, Shimosato T, Sakonjo H, Miyazaki M. Candesartan and amlodipine combination therapy provides powerful vascular protection in stroke-prone spontaneously hypertensive rats. Hypertens Res 2011; 34: 245-252.

6 Hayashi K, Wakino S, Sugano N, Ozawa Y, Homma K, Saruta T. $\mathrm{Ca}^{2+}$ channel subtypes and pharmacology in the kidney. Circ Res 2007; 100: 342-353.

7 Fujisawa T, Ikegami H, Noso S, Hiromine Y, Kawabata Y, Nishino M, Asano K, Ogihara $\mathrm{T}$. Renoprotective effect of $\mathrm{N}$-type $\mathrm{Ca}$ channel blockade in diabetic nephropathy. Diabetes Complications 2007; 21: 252-257.

8 Toba H, Yoshida M, Tojo C, Nakano A, Oshima Y, Kojima Y, Noda K, Wang J, Kobara M, Nakata T. L/N-type calcium channel blocker cilnidipine ameliorates proteinuria and inhibits the renal renin-angiotensin-aldosterone system in deoxycorticosterone acetatesalt hypertensive rats. Hypertens Res 2011; 34: 521-529.

9 Honda M, Hayashi K, Matsuda H, Kubota E, Tokuyama H, Okubo K, Takamatsu I, Ozawa Y, Saruta T. Divergent renal vasodilator action of L- and T-type calcium antagonists in vivo. J Hypertens 2001; 19: 2031-2037.

10 Takai S, Jin D, Sakonjo H, Miyazaki M. Combination therapy with irbesartan and efonidipine for attenuation of proteinuria in Dahl salt-sensitive rats. Hypertens Res 2010; 33: 953-959.

11 Hirning LD, Fox AP, McCleskey EW, Olivera BM, Thayer SA, Miller RJ, Tsien RW Dominant role of $\mathrm{N}$-type $\mathrm{Ca}^{2}+$ channels in evoked release of noradrenaline from sympathetic neurons. Science 1988; 239: 57-61.

12 Morimoto S, Yano Y, Maki K, Iwasaka T. Renal and vascular protective effects of cilnidipine in patients with essential hypertension. J Hypertens 2007; 25 2178-2183.

13 Takai S, Jin D, Ikeda H, Sakonjo H, Miyazaki M. Significance of angiotensin II receptor blockers with high affinity to angiotensin II type 1 receptors for vascular protection in rats. Hypertens Res 2009; 32: 853-860.

14 Takai S, Jin D, Miyazaki M. Irbesartan prevents metabolic syndrome in rats via activation of peroxisome proliferator-activated receptor $\gamma$. J Pharmacol Sci 2011; 116 309-315.

15 Dong YF, Kataoka K, Tokutomi Y, Nako H, Nakamura T, Toyama K, Sueta D, Koibuchi N, Yamamoto E, Ogawa H, Kim-Mitsuyama S. Beneficial effects of combination of valsartan and amlodipine on salt-induced brain injury in hypertensive rats. J Pharmacol Exp Ther 2011; 339: 358-866.

16 Kerr S, Brosnan MJ, McIntyre M, Reid JL, Dominiczak AF, Hamilton CA. Superoxide anion production is increased in a model of genetic hypertension: role of the endothelium. Hypertension 1999; 33: 1353-1358.

17 Brosnan MJ, Hamilton CA, Graham D, Lygate CA, Jardine E, Dominiczak AF. Irbesartan lowers superoxide levels and increases nitric oxide bioavailability in blood vessels from spontaneously hypertensive stroke-prone rats. J Hypertens 2002; 20: 281-286.

18 Takai S, Kirimura K, Jin D, Muramatsu M, Yoshikawa K, Mino Y, Miyazaki M. Significance of angiotensin II receptor blocker lipophilicities and their protective effect against vascular remodeling. Hypertens Res 2005; 28: 593-600.

19 Seshiah PN, Weber DS, Rocic P, Valppu L, Taniyama Y, Griendling KK. Angiotensin II stimulation of $\mathrm{NAD}(\mathrm{P}) \mathrm{H}$ oxidase activity: upstream mediators. Circ Res 2002; 91: 406-413.

20 Fukui T, Ishizaka N, Rajagopalan S, Laursen JB, Capers 4th Q, Taylor WR, Harrison DG, de Leon H, Wilcox JN, Griendling KK. p22 ${ }^{\text {phox }}$ mRNA expression and NADPH oxidase activity are increased in aortas from hypertensive rats. Circ Res 1997; 80: 45-51.

21 Hamilton CA, Brosnan MJ, Al-Benna S, Berg G, Dominiczak AF. NAD(P)H oxidase inhibition improves endothelial function in rat and human blood vessels. Hypertension 2002; 40: 755-762.

22 Hishikawa K, Takase O, Idei M, Fujita T. Comparison of antioxidant activity of cilnidipine and amlodipine. Kidney Int 2009; 76: 230-231.

23 Lee YJ, Park KH, Park HH, Kim YJ, Lee KY, Kim SH, Koh SH. Cilnidipine mediates a neuroprotective effect by scavenging free radicals and activating the phosphatidylinositol 3-kinase pathway. J Neurochem 2009; 111: 90-100.

24 Macarthur H, Westfall TC, Wilken GH. Oxidative stress attenuates NO-induced modulation of sympathetic neurotransmission in the mesenteric arterial bed of spontaneously hypertensive rats. Am J Physiol Heart Circ Physiol 2008; 294: H183-H189.

25 Miyagawa K, Ohashi M, Yamashita S, Kojima M, Sato K, Ueda R, Dohi Y. Increased oxidative stress impairs endothelial modulation of contractions in arteries from spontaneously hypertensive rats. J Hypertens 2007; 25: 415-421.

26 Brosnihihan KB, Li P, Ferrario CM. Angiotensin-(1-7) dilates canine coronary arteries through kinins and nitric oxide. Hypertension 1996; 27: 523-528.

27 Donoghue M, Hsieh F, Baronas E, Godbout K, Gosselin M, Stagliano N, Donovan M, Woolf $B$, Robison K, Jeyaseelan R, Breitbart RE, Acton S. A novel angiotensinconverting enzyme-related carboxypeptidase (ACE2) converts angiotensin I to angiotensin 1-9. Circ Res 2000; 87: e1-e9.

28 Xu P, Costa-Goncalves AC, Todiras M, Rabelo LA, Sampaio WO, Moura MM, Santos SS, Luft FC, Bader M, Gross V, Alenina N, Santos RA. Endothelial dysfunction and elevated blood pressure in Mas gene-deleted mice. Hypertension 2008; 51: 574-580.

29 Rabelo LA, Alenina N, Bader M. ACE2-angiotensin-(1-7)-Mas axis and oxidative stress in cardiovascular disease. Hypertens Res 2011; 34: 154-160.

30 Ishiyama Y, Gallagher PE, Averill DB, Tallant EA, Brosnihan KB, Ferrario CM. Upregulation of angiotensin-converting enzyme 2 after myocardial infarction by blockade of angiotensin II receptors. Hypertension 2004; 43: 970-976.

31 Igase M, Strawn WB, Gallagher PE, Geary RL, Ferrario CM. Angiotensin II AT 1 receptors regulate ACE2 and angiotensin-(1-7) expression in the aorta of spontaneously hypertensive rats. Am J Physiol Heart Circ Physiol 2005; 289: H1013-H1019.

32 Konda T, Enomoto A, Aritomi S, Niinuma K, Koganei H, Ogawa T, Nitta K. Different effects of L/N-type and L-type calcium channel blockers on the renin-angiotensinaldosterone system in SHR/Izm. Am J Nephrol 2009; 30: 155-161.

33 Aritomi S, Niinuma K, Ogawa T, Konda T, Nitta K. Effects of an N-type calcium antagonist on angiotensin II-renin feedback. Am J Nephrol 2011; 33: 168-175.

34 Tom B, de Vries R, Saxena PR, Danser AH. Bradykinin potentiation by angiotensin-(17) and ACE inhibitors correlates with ACE C- and N-domain blockade. Hypertension 2001; 38: 95-99.

35 Fan L, Yang Q, Xiao XQ, Grove KL, Huang Y, Chen ZW, Furnary A, He GW. Dual actions of cilnidipine in human internal thoracic artery: inhibition of calcium channels and enhancement of endothelial nitric oxide synthase. J Thorac Cardiovasc 2011; 141 1063-1069.

36 Sharma A, Trane A, Yu C, Jasmin JF, Bernatchez P. Amlodipine increases endothelial nitric oxide release by modulating binding of native eNOS protein complex to caveolin1. Eur J Pharmacol 2011; 659: 206-212.

37 Takami T, Shigemasa M. Efficacy of various antihypertensive agents as evaluated by indices of vascular stiffness in elderly hypertensive patients. Hypertens Res 2003; 26 609-614.
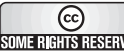
License. To view a copy of this license, visit http://creativecommons.org/ licenses/by-nc-sa/3.0/

This work is licensed under the Creative Commons Attribution-NonCommercial-Share Alike 3.0 Unported 